HUB-EP-97/36

hep-th/9706093

\title{
Three-Branes and Five-Branes in $N=1$ Dual String Pairs
}

\author{
Björn Andreas and Gottfried Curio円 \\ Humboldt-Universität zu Berlin, Institut für Physik, D-10115 Berlin, Germany
}

\begin{abstract}
In this note we show that in dual $N=1$ string vacua provided by the heterotic string on an elliptic Calabi-Yau together with a vector bundle respectively $F$-theory on Calabi-Yau fourfold the number of heterotic fivebranes necessary for anomaly cancellation matches the number of $F$-theory threebranes necessary for tadpole cancellation. This extends to the general case the work of Friedman, Morgan and Witten, who treated the case of embedding a heterotic $E_{8} \times E_{8}$ bundle, leaving no unbroken gauge group, where one has a smooth Weierstrass model on the $F$-theory side.
\end{abstract}

\footnotetext{
${ }^{1}$ email: andreas@qft3.physik.hu-berlin.de, curio@qft2.physik.hu-berlin.de
} 
In the course of study of $F$-theory compactifications with $N=1$ supersymmetry in four dimensions on a Calabi-Yau fourfold $X$ there was established the necessity of turning on a number $n_{3}=\chi(X) / 24$ of spacetime-filling threebranes for tadpole cancellation [3]. This should be compared with a potentially dual heterotic compactification on an elliptic Calabi-Yau $Z$ with vector bundle $V$ embedded in $E_{8} \times E_{8}$ got by adiabtic extension over a common (complex) twofold base $B$ of the eight-dimensional duality of $F$-theory on $K 3$ with the heterotic string on $T^{2}$ [1]. There the threebranes should correspond to a number $n_{5}$ of fivebranes wrapping the elliptic fibre. Their necessity for a consistent heterotic compactification (independent of any duality considerations) was established in the exhaustive study done by Friedman, Morgan and Witten on vector budles and $F$-theory [2]. There it was also shown that in the case of an $E_{8} \times E_{8}$ vector bundle $V$, leaving no unbroken gauge group and corresponding to a smooth Weierstrass model for the fourfold, it is possible to express $n_{3}$ and $n_{5}$ in comparable and indeed matching data on the common base $B$. This matching will here be extended to the general case.

To achieve this we will adopt a somewhat different technical procedure. In the case of not having a smooth Weierstrass model there occured already in [3] the difficulty to reduce the fourfold expression $\chi(X) / 24$ for $n_{3}$ to an expression involving only suitable data of the base $B^{3}$ of the elliptic $F$-theory fibration of $X$ (not to be confused with the twofold base $B^{2}$ of the $K 3$ fibration of $X$, here denoted simply by $B$, which is visible also on the heterotic side), which was [2] only an intermediate step to reduce the expression to one involving only twofold base data. For this reason we express here $\chi(X)$ directly in the Hodge numbers of $X$ and match then these with the data of the dual heterotic model; here essential use is made of an index-formula computation, also initiated in [2], to describe the moduli of the bundle $V$.

In the course of the actual computation we will assume that $V$ is a $S U\left(n_{1}\right) \times S U\left(n_{2}\right)$ bundle. Furthermore we will implement the adiabatically extended duality by the following specification [2]: as $X$ is assumed to be a $K 3$ fibration over $B$ it follows that $B^{3}$, the threefold base of the $F$-theory elliptic fibration, is a $P^{1}$ fibration over $B$; this fibration structure is described by assuming the $P^{1}$ bundle over $B$ to be a projectivization of a vector bundle $Y=\mathcal{O} \oplus \mathcal{T}$, with $\mathcal{T}$ a line bundle over $B$; then the cohomology class $t=c_{1}(\mathcal{T})$ encodes the $P^{1}$ fibration structure. Now the duality is implemented by choosing our $S U\left(n_{1}\right) \times S U\left(n_{2}\right)$ bundle so that

$$
\eta_{1}=\pi_{*}\left(c_{2}\left(V_{1}\right)\right)=6 c_{1}(B)+t, \quad \eta_{2}=\pi_{*}\left(c_{2}\left(V_{2}\right)\right)=6 c_{1}(B)-t
$$

Now let us start and first compute the number of heterotic five-branes $n_{5}$ using the non-perturbative anomaly cancellation condition which is given by [2]

$$
c_{2}\left(V_{1}\right)+c_{2}\left(V_{2}\right)+[W]=c_{2}(T Z),
$$

where $T Z$ is tangent bundle of the Calabi-Yau threefold $Z$ on which the heterotic string is compactified; $c_{2}(T Z)$ was derived in [2] (we use the notation $c_{i}=c_{i}(B)$ and $\sigma$ for the

\footnotetext{
${ }^{2}$ Note that in case some of the threebranes have dissolved into finite-sized instantons in the worldvolume gauge theory of the $F$-theory sevenbrane this is accompanied by a corresponding fivebrane transition on the heterotic side [8, 2, 计.
} 
class of a section of $\pi$ )

$$
c_{2}(T Z)=c_{2}+11 c_{1}^{2}+12 \sigma c_{1}
$$

(here on the right hand side the classes represent their pullbacks to $Z$ ). Furthermore $[W]$ is the cohomology class of the five-branes where $[W]=n_{5}[F]=\pi^{*}([p])$ and $[F]$ is the class in $H^{4}$ of the fiber of the elliptic fibration.

For explicit evaluation and to establish some notation we give here the actual number of fivebranes for $V$ a $S U\left(n_{1}\right) \times S U\left(n_{2}\right)$ bundle. The second Chern class for a $S U(n)$ bundle is given by (with $\eta=\pi_{*}\left(c_{2}(V)\right)$ and $\mathcal{L}$ being some line bundle over $B$ ) [2]

$$
c_{2}(V)=\eta \sigma-\frac{1}{24} c_{1}(\mathcal{L})^{2}\left(n^{3}-n\right)-\frac{n}{8} \eta\left(\eta-n c_{1}(\mathcal{L})\right)
$$

Using the above relations for $\eta_{1}, \eta_{2}$ and $c_{2}(V)$ we can derive (where also $c_{1}(B)=c_{1}(\mathcal{L})$ by the Calabi-Yau condition for $Z$ )

$$
c_{2}\left(V_{1 / 2}\right)=6 c_{1} \sigma \pm t \sigma-\frac{1}{24} c_{1}^{2}\left(n_{1}^{3}-n_{1}\right)-\frac{n_{1}}{8}\left[36 c_{1}^{2} \pm 12 c_{1} t+t^{2}-6 n_{1} c_{1}^{2} \mp n_{1} t c_{1}\right]
$$

We find for the number of five branes

$$
\begin{aligned}
n_{5}= & c_{2}+11 c_{1}^{2}+\frac{1}{24} c_{1}^{2}\left(n_{1}^{3}-n_{1}+n_{2}^{3}-n_{2}\right)+\frac{\left(n_{1}+n_{2}\right)}{4}\left(18 c_{1}^{2}+\frac{t^{2}}{2}\right)+ \\
& +\frac{\left(n_{1}-n_{2}\right)}{4} 6 c_{1} t-\frac{\left(n_{1}^{2}+n_{2}^{2}\right)}{8} 6 c_{1}^{2}+\frac{\left(n_{2}^{2}-n_{1}^{2}\right)}{8} t c_{1}
\end{aligned}
$$

Now let us express the number of $F$-theory 3-branes $n_{3}=\chi(X) / 24$ in terms of heterotic data. Because of $\chi(X) / 6-8=h^{1,1}(X)+h^{3,1}(X)-h^{2,1}(X)$ (cf. [3]) we have to use the following informations ${ }^{3}$

$$
\begin{aligned}
& h^{1,1}(X)=h^{1,1}(Z)+1+r=12-c_{1}^{2}+r \\
& h^{2,1}(X)=n_{o} \\
& h^{3,1}(X)=h^{2,1}(Z)+I+n_{o}+1=12+29 c_{1}^{2}+I+h^{2,1}(X)
\end{aligned}
$$

where in the first line we used Noethers formula $1=\frac{c_{1}^{2}(B)+c_{2}(B)}{12}$ and $\chi(Z)=-60 \int_{B} c_{1}^{2}(B)$ [5]; for the $n_{o}$ in the second line see below. Let us now see how the expression for $h^{3,1}(X)$ emerges. Note that the moduli space $\mathcal{M}$ for bundles on $Z$ of dimension $m_{\text {bun }}$ has a fibration $\mathcal{M} \rightarrow \mathcal{Y}$ which corresponds on the $F$-theory side to a fibration of the abelian

\footnotetext{
${ }^{3}$ We assume that the elliptic fibration $Z \rightarrow B$ has only one section so that $h^{1,1}(Z)=h^{1,1}(B)+1=$ $c_{2}(B)-1=11-c_{1}^{2}(B)$; let us furthermore assume that no fourflux [6] is turned on; $r$ denotes the rank of the unbroken non-abelian gauge group (do not confuse it with the rank of the group of the bundle $V$ ); we furthermore assume that we have no further $U(1)$ factors (coming from sections).
} 
variety $H^{3}(X, \mathbf{R}) / H^{3}(X, \mathbf{Z})$ of complex dimension $h^{2,1}(X)$ over a part of the space of complex deformations of $X$ (cf. [2]); the remaining complex deformations account for the complex deformations of the heterotic Calabi-Yau $(+1)$, i.e.

$$
h^{3,1}(X)+h^{2,1}(X)=h^{2,1}(Z)+m_{\text {bun }}+1
$$

Now concerning the contribution of the bundle moduli let us recall the setup of the index-computation in [2]. As the usual quantity suitable for index-computation $\sum_{i=o}^{3}(-1)^{i} h^{i}(Z, a d(V))$ vanishes by Serre duality one has to introduce a further twist and to compute a character-valued index. Now because of the elliptic fibration structure one has on $Z$ the involution $\tau$ coming from the "sign-flip" in the fibers which we furthermore assume has been lifted to an action on the bundle. The character-valued index

$$
I=-\frac{1}{2} \sum_{i=o}^{3}(-1)^{i} \operatorname{Tr}_{H^{i}(Z, a d(V))} \tau
$$

simplifies by the vanishing of the ordinary index to

$$
I=-\sum_{i=o}^{3}(-1)^{i} h^{i}(Z, a d(V))_{e}
$$

where the subscript "e" (resp. "o") indicates the even (resp. odd) part. As we have an unbroken gauge group $H$, which is the commutator of the group $G$ of $V$, one finds $I=n_{e}-n_{o}$ corrected by $h_{e}^{0}-h_{o}^{0}$ denoting by $n_{e / o}$ the number $h^{1}(Z, a d(V))_{e / o}$ of massless even/odd chiral superfields and by $h_{e / o}^{0}$ the number of unbroken gauge group generators even/odd under $\tau$. The unbroken gauge group is in this $n_{3^{-}}$calculation accounted for by the rank contribution in $h^{1,1}(X)$ for the resolved fourfold.

So one has for the number of bundle moduli $m_{\text {bun }}=h^{1}(Z, a d(V))=n_{e}+n_{o}=I+2 n_{o}$ and so gets the announced expression for $h^{3,1}(X)$. Furthermore on the $F$-theory side the modes odd under the involution $\tau^{\prime}$ corresponding to the heterotic involution $\tau$ correspond to the $h^{2,1}(X)$ classes [2] (we assume no four-flux was turned on).

Now let us go to the index-formula for a $S U\left(n_{1}\right) \times S U\left(n_{2}\right)$ bundle. The index for $S U(n)$ is given by [2]

$$
I=n-1-\int_{U_{1}} c_{2}(V)-\int_{U_{2}} c_{2}(V)
$$

Recall the $U_{i}$ are the components of the fixed point set of the involution $\tau$ where $U_{1}$ is the class of the section $\sigma$ of $Z \rightarrow B$ and $U_{2}$ is a triple cover of $B$ which has cohomology class $3 \sigma+3 c_{1}(\mathcal{L})$. Let us go on and express this purely in cohomology of $B$. This can be done in two ways. First one can use the identity $\int_{U_{i}} c_{2}(V)=\left.\int_{B} c_{2}(V)\right|_{U_{1}}+\left.3 \int_{B} c_{2}(V)\right|_{U_{2}}$ where the 3 appears since $U_{2}$ is a triple cover of $B$ and restricts the non-perturbative anomaly condition to the fixed point set (cf. [7]). The restricted second Chern-classes of 
$\left.T Z\right|_{U_{i}}$ were direved in [7] as $c_{2}\left(\left.T Z\right|_{U_{1}}\right)=c_{2}-c_{1}^{2}$ and $c_{2}\left(\left.T Z\right|_{U_{2}}\right)=c_{2}+11 c_{1}^{2}$. Thus for the index we find (denoting $r k=n_{1}+n_{2}-2=16-r$ ))

$$
I=r k-\left(c_{2}-c_{1}^{2}-n_{5}+3 c_{2}+33 c_{1}^{2}-3 n_{5}\right)=r k-\left(48+28 c_{1}^{2}\right)+4 n_{5}
$$

Alternatively one can use formula for the restriction of $c_{2}(V)$ to the fixed point set given in [2]

$$
\begin{aligned}
I & =n-1+\int_{B}\left(\frac{\left(n^{3}-n\right) c_{1}(\mathcal{L})^{2}}{6}+\frac{n \eta\left(\eta-n c_{1}(\mathcal{L})\right)}{2}+\eta c_{1}(\mathcal{L})\right) \\
& =n-1-4\left(c_{2}-\eta \sigma\right)+\eta c_{1}
\end{aligned}
$$

giving again for the total index $I=I_{1}+I_{2}$ the result

$$
\begin{aligned}
I & =r k-4\left(c_{2}\left(V_{1}\right)+c_{2}\left(V_{2}\right)\right)+48 c_{1} \sigma+12 c_{1}^{2} \\
& =r k-4\left(c_{2}+11 c_{1}^{2}+12 \sigma c_{1}\right)+4 n_{5}+48 c_{1} \sigma+12 c_{1}^{2} \\
& =r k-\left(48+28 c_{1}^{2}\right)+4 n_{5}
\end{aligned}
$$

One finally gets then with $r=16-r k$ and our expression for $I$ that

$$
\chi(X) / 24=2+\frac{1}{4}\left(12-c_{1}^{2}+16-r k+12+29 c_{1}^{2}+I\right)=n_{5}
$$

matching the heterotic value.

\section{References}

1. C. Vafa, Evidence for F-theory, Nucl. Phys. B 469 (1996) 493, hep-th/9602022.

2. R. Friedman, J. Morgan and E. Witten, Vector Bundles and F-Theory, hepth/9701162.

3. S. Sethi, C. Vafa and E. Witten, Constraints on Low-Dimensional String Compactifications, Nucl. Phys. B 480 (1996) 213, hep-th/9606122.

4. E. Sharpe, Extremal Transitions in Heterotic String Theory, hep-th/9705210.

5. A. Klemm, B. Lian, S.-S. Roan and S.-T. Yau, Calabi-Yau fourfolds for $M$ - and F-Theory Compactifications, hep-th/9701023.

6. E. Witten, On Flux Quantization in M-Theory and the Effective Action, hepth/9609122. 
7. B. Andreas, G. Curio and D. Lüst, $N=1$ Dual String Pairs and their Massless Spectra, hep-th/9705174.

8. M. Bershadsky, A. Johansen, T. Pantev and V. Sadov, On Four-Dimensional Compactifications of F-Theory, hep-th/9701165. 